\title{
The Presynaptic Dense Projection of the Caenorhabiditis elegans Cholinergic Neuromuscular Junction Localizes Synaptic Vesicles at the Active Zone through SYD-2/Liprin and UNC-10/RIM-Dependent Interactions
}

\author{
Christian Stigloher, ${ }^{1,2,3}$ Hong Zhan, ${ }^{1,2,3}$ Mei Zhen, ${ }^{4}$ Janet Richmond, ${ }^{5}$ and Jean-Louis Bessereau ${ }^{1,2,3}$ \\ ${ }^{1}$ Institut de Biologie de l'École Normale Supérieure, Biology Department, F-75005 Paris, France, ${ }^{2}$ Inserm, Unité 1024, F-75005 Paris, France, ${ }^{3}$ Centre \\ National de la Recherche Scientifique, Unité Mixte de Recherche 8197, F-75005 Paris, France, ${ }^{4}$ Samuel Lunenfeld Research Institute, University of Toronto, \\ Ontario, Canada, M5G 1X5, and 5Department of Biological Sciences, University of Illinois, Chicago, Illinois 60607
}

The active zone (AZ) of chemical synapses is a specialized area of the presynaptic bouton in which vesicles fuse with the plasma membrane and release neurotransmitters. Efficient signaling requires synaptic vesicles (SVs) to be recruited, primed, and retained at the AZ, in close proximity to voltage-dependent calcium channels that are activated during presynaptic depolarization. The electron-dense specializations at the $\mathrm{AZ}$ might provide a molecular platform for the spatial coordination of these different processes. To investigate this hypothesis, we examined high-resolution three-dimensional models of Caenorhabditis elegans cholinergic neuromuscular junctions generated by electron tomography. First, we found that SVs are interconnected within the bouton by filaments similar to those described in vertebrates. Second, we resolved the three-dimensional structure of the dense projection centered in the AZ. The dense projection is a more complex structure than previously anticipated, with filaments radiating from a core structure that directly contact SVs in the interior of the bouton as well as SVs docked at the plasma membrane. Third, we investigated the functional correlate of these contacts by analyzing mutants disrupting two key AZ proteins: UNC-10/RIM and SYD-2/liprin. In both mutants, the number of contacts between SVs and the dense projection was significantly reduced. Similar to unc-10 mutants, the dependence of SV fusion on extracellular calcium concentration was exacerbated in syd-2 mutants when compared with the wild type. Hence, we propose that the dense projection ensures proper coupling of primed vesicles with calcium signaling by retaining them at the AZ via UNC-10/RIM and SYD-2/liprindependent mechanisms.

\section{Introduction}

The spatial compartmentalization of synaptic boutons is essential to retain, release, and recycle synaptic vesicles (SVs). Fusion of most synaptic vesicles occurs within a specific region of the bouton called the active zone (AZ). At the morphological level, the $\mathrm{AZ}$ is identified by the presence of SVs docked to the plasma membrane near an electron-dense presynaptic structure (Gray, 1963; Couteaux and Pécot-Dechavassine, 1970; Zhai and Bellen,

\footnotetext{
Received Nov. 25, 2010; revised Jan. 14, 2011; accepted Jan. 21, 2011.

This work was funded by Inserm, Agence Nationale de la Recherche Grant ANR-07-NEURO-032-01, the Fondation pour la Recherche Medicale, Fédération pour la Recherche sur le Cerveau, Rotary International, and the Association Française contre les Myopathies. C.S. was supported by a fellowship within the Postdoctoral Program of the German Academic Exchange Service, the European Molecular Biology Organization, and the International Human Frontier Science Program Organization. J.R. was supported by National Institutes of Health Grant R01 MH073156. M.Z. was supported by Canadian Institute of Health Research Grant MOP 209314. We thank Serge Marty for critical reading of this manuscript, Fabrice Richard for TEM micrographs, Wesley Hung for strains, the ENS EM platform (Philippe Rostaing and Laure Wingertsmann), the Service de Microscopie Electronique Institut Fédératif de Recherche 83 at University Pierre and Marie Curie (Jean-Pierre Lechaire and Ghislaine Frebourg), and the Advanced Bioimaging Centre, Mount Sinai Hospital, Toronto (Douglas Holmyard) for access to sample preparation devices, electron microscopes, and technical support.

Correspondence should be addressed to Jean-Louis Bessereau, Institute of Biology of the École Normale Supérieure, 46 rue d'Ulm, Paris, F-75005 France. E-mail: jlbesse@biologie.ens.fr.

DOI:10.1523/JNEUROSCI.6164-10.2011

Copyright $\odot 2011$ the authors $\quad 0270-6474 / 11 / 314388-09 \$ 15.00 / 0$
}

2004; Siksou et al., 2009). Functionally, the AZ represents a domain in which primed vesicles fuse in response to a local calcium influx triggered by presynaptic depolarization (Sudhof, 2004). The molecular basis for these morphological and functional AZ features remains to be fully elucidated. Numerous proteins have been identified within the cytomatrix at the active zone (CAZ). The best-characterized CAZ components are the proteins regulating SV fusion, including RIM, Munc13, and the SNARE complex, for which electrophysiological analyses and biochemical assays provided quantitative functional readouts. A second class of proteins, including Bassoon, Piccolo, CAST/ERK/ELKS, and liprin- $\alpha$, are thought to be structural components of the presynaptic specialization and its associated cytoskeleton (for review, see Schoch and Gundelfinger, 2006; Jin and Garner, 2008). All of these proteins contain multiple protein-protein interaction domains that allow physical interplay between release factors and structural components. For example, Munc13-1 can form heteromeric complexes with Piccolo, Bassoon, CAST1, and Rim1 (Wang et al., 2009) and also assembles into a trimeric complex with RIM $2 \alpha$ and Rab3A (Dulubova et al., 2005). Deciphering the functional relevance of these protein networks in a cellular context remains a challenge. 
Here we use the Caenorhabditis elegans neuromuscular junction (NMJ) to evaluate the interplay between the SV release machinery and the dense projection, which provides a landmark to analyze the distribution of SVs and AZ components (White et al., 1986). By immunoelectron microscopy, SYD-2/liprin is detected within the dense projection (Yeh et al., 2005), whereas UNC-10/ RIM and UNC-13 are enriched at the plasma membrane within $100 \mathrm{~nm}$ of the dense projection (Weimer et al., 2006), suggesting that this area corresponds to the AZ. Consequently, UNC-13dependent "docked" vesicles contacting the plasma membrane are primarily confined to this same region. Disrupting unc-10 causes a redistribution of the docked SVs away from the dense projection (Weimer et al., 2006). These results suggested that the dense projection might retain docked SVs at the AZ. However, evidence demonstrating a physical link between the dense projection and SVs within C. elegans AZs has not yet been determined because of three-dimensional (3D) resolution limits of conventional electron microscopy (EM).

Here we applied electron tomography to adult C. elegans, preserved by high-pressure freeze fixation (HPF), to obtain high-resolution 3D models of the presynaptic ultrastructure (McDonald and Auer, 2006; Bárcena and Koster, 2009). First, we observed a presynaptic cytomatrix connecting SVs. Second, our $3 \mathrm{D}$ reconstruction of the dense projection revealed an elaborate structure, from which emanating filaments contact both docked and undocked SVs. Third, the analysis of syd-2/liprin and unc-10/ RIM mutants by this methodology led to the identification of a novel mechanism to retain SVs at the AZ and ensure efficient coupling with calcium signaling.

\section{Materials and Methods}

C. elegans genetics. N2 Bristol (also referred to as wild type in this study), unc-10(md1117) (Koushika et al., 2001), syd-2(ju37) (Zhen and Jin, 1999), and syd-2(ju37) unc-10(md1117) were used in this study. syd2(ju37) unc-10(md1117) double mutants were generated by intercrossing syd-2(ju37) males with unc-10( $m$ d1117) hermaphrodites. F1 showing a wild-type-like movement phenotype were picked, and F2 offspring with a syd-2 phenotype were isolated and cloned. F3 with a coiler phenotype were isolated as putative syd-2 unc-10 double mutants and confirmed by genotyping in F4.

HPF and freeze substitution of C. elegans. High-pressure freezing and freeze substitution was conducted as described previously for morphometric analysis (Weimer, 2006) with modifications. In short, $\sim 10$ young adult hermaphrodites were loaded into the $100 \mu \mathrm{m}$ deep well of the freezing chamber [Specimen Carriers Type A $(100 \mu \mathrm{m})$ and B $(0 \mu \mathrm{m})$; Bal-Tec AG, acquired by Leica Microsystems] filled with a mixture of $10 \%$ BSA and pelleted OP50 bacteria (ratio, 20:100, v/v) and processed by an EM HPM100 (Leica Microsystems) at a freezing speed $>20,000 \mathrm{~K} / \mathrm{s}$ and a pressure $>2000$ bar. High-pressure frozen worm/bacteria pellets were directly transferred in liquid nitrogen to an EM AFS2 freeze substitution system (Leica Microsystems). Freeze chambers were opened to allow better penetration during freeze substitution. Samples were incubated in $0.1 \%$ tannic acid and $0.5 \%$ glutaraldehyde in anhydrous acetone at $-90^{\circ} \mathrm{C}$ for $96 \mathrm{~h}$, washed four times for $1 \mathrm{~h}$ with anhydrous acetone at $-90^{\circ} \mathrm{C}$, and fixed in $2 \% \mathrm{OsO}_{4}$ in anhydrous acetone at $-90^{\circ} \mathrm{C}$ for $28 \mathrm{~h}$, and then temperature was ramped for $14 \mathrm{~h}$ to $-20^{\circ} \mathrm{C}$, incubated $16 \mathrm{~h}$ at $-20^{\circ} \mathrm{C}$, ramped again to $4^{\circ} \mathrm{C}$ in $4 \mathrm{~h}$, and immediately washed with anhydrous acetone at $4^{\circ} \mathrm{C}$ four times at $0.5 \mathrm{~h}$ intervals. Then the temperature was gradually raised to $20^{\circ} \mathrm{C}$ in $1 \mathrm{~h}$, and samples were subsequently transferred for embedding in a series of freshly prepared Araldite solutions at increasing concentrations (50\% Araldite in acetone for $3 \mathrm{~h}$ at room temperature, $90 \%$ Araldite in acetone overnight at $4^{\circ} \mathrm{C}$, followed by 2 times pure Araldite at room temperature). Araldite infiltrated samples were polymerized for $48 \mathrm{~h}$ at $60^{\circ} \mathrm{C}$.

EM section preparations. Serial sections, $250 \mathrm{~nm}$, were cut and positioned onto $50 \mathrm{~nm}$ LUXFilm-coated slotted grids (12814-CU; Ted Pella) and contrasted with $2 \%$ uranyl acetate and Reynolds lead citrate. For fiducial-aided image alignment, $10 \mathrm{~nm}$ ProtA-Au-beads (EM.PAG10; BB International) were added to both sides of the sample by bathing in undiluted solution for $20 \mathrm{~min}$ and subsequent washing. A 5-7 nm carbon coat was applied to the sample to improve sample stability under the electron beam. Potential ice crystal formation in samples was evaluated by surveying the preservation of cell nuclei in which ice formation is most apparent and generates compaction artifacts. Corresponding samples were excluded from additional analysis.

Electron tomography. Tilt image acquisition was conducted from $-60^{\circ}$ to $+60^{\circ}$ with $1^{\circ}$ increments at $200 \mathrm{kV}$ with a JEOL $2100 \mathrm{HC}$ electron microscope equipped with a GATAN GIF863 Tridiem post column energy filter and a GATAN camera with $2048 \times 2048$ pixel resolution. To reduce noise attributable to the image acquisition process, Gaussian Blur filtering was conducted on raw images with the NIH ImageJ Gaussian Blur Filter Tool (Wayne S. Rasband, National Institutes of Health, Bethesda, MD; http://rsb.info.nih.gov/ij/) set to a "Sigma (radius)" value of 1.0. For tilt-image series alignment and tomographic reconstruction, the ETomo/IMOD software package was used with fiducial-guided or alternatively fiducial-less alignment modes (Kremer et al., 1996). Pixel size of the tilt series acquisition images is $0.47 \mathrm{~nm}$ per pixel. To reduce noise attributable to tomogram generation, bandpass filtering of the generated tomogram image stack was performed using the NIH ImageJ Band Pass Filter tool with the parameters lower cutoff at 1 pixel and the upper cutoff at 100 pixels with $5 \%$ tolerance of direction. Tomogram resolution was estimated according to Zampighi et al. $(2005,2008)$ with the measurement of the "unit membrane" thickness (Robertson, 1987). The resulting estimation of tomogram resolution ranges from 4 to $5 \mathrm{~nm}$.

Image segmentation and quantification. For dense projection and docked vesicle quantifications, 15 tomograms were segmented and quantified [five for each genotype: unc-10(md1117), syd-2(ju37) and N2]. For the detailed vesicle, filament, and connectivity analysis, the cytomatrix filaments of two N2 tomograms were segmented exhaustively $(n=117$ filaments), and the corresponding synaptic vesicle diameter and connectivity were annotated and measured individually $(n=263$ SVs). To appreciate the full extent of the dense projection, three consecutive tomograms of 250-nm-thick sections of a N2 sample were aligned using the "join" function of the ETomo software package without previous stretching or compression. Image segmentation and quantification was conducted with Amira 4.1.1 (Mercury Computer Systems). To annotate dense projections, an Amira "labelfield" was defined, and densities were selected with a region growing tool. Gray value threshold was adjusted to mark the whole extent of the densities. These steps were repeated for every single virtual slice section of the entire tomogram stack. Filaments interconnecting synaptic vesicles were annotated in a similar manner. Cell membranes and outer mitochondrial membranes were segmented manually every 5 th or 10 th virtual tomogram section to provide a spatial view without obscuring other cellular structures. Microtubules were segmented manually with a circle tool set fitting to the microtubule thickness. Synaptic vesicles were annotated with the "landmark tool" available in the Amira software package. The spherical landmark was centered in the middle of the tomogram structure of the vesicle and color coded in yellow. Diameters were either adjusted to an average vesicle diameter or, in the case of the filament and vesicle length quantification experiments, adjusted to the actual radius of every vesicle. Docked vesicles were annotated accordingly but as a separate landmark object group with red spheres. The docked state of SVs was defined by direct contact between the membrane of the vesicle and the plasma membrane. For the distribution analysis of docked vesicles overall, 158 docked vesicles were annotated in 15 tomograms. [N2, $n=58$ in 5 tomograms/synapses; unc10(md1117), $n=54$ in 5 tomograms/synapses; syd-2(ju37), $n=46$ in 5 tomograms/synapses]. Vesicles that were not docked but contacted by the dense projection were annotated in a third landmark group in orange. For the analysis of nondocked vesicles contacting the dense projection overall, 61 vesicles were annotated in 15 tomograms [N2, $n=35$ in 5 tomograms/ synapses; unc-10(md1117), $n=12$ in 5 tomograms/synapses; syd-2(ju37), $n=14$ in 5 tomograms/synapses]. Dense-core vesicles were annotated correspondingly in a fourth landmark group in blue. 
Distances were measured in three dimensions using the "3D measurement tool" of Amira. For quantification of distances between docked vesicles and dense projections, the shortest distance to the closest process of the dense projection was assessed. The segmentations and landmarks were tilted in 3D to define the shortest distance in space. Similarly, filament lengths were measured in 3D.

Statistical analysis. The Kolmogorov-Smirnov test (KS test) was computed online (www. physics.csbsju.edu/stats/KS-test.html). The R-software package (R Foundation for Statistical Computing) and Prism (GraphPad Software) were used for other statistical tests.

Electrophysiology. Electrophysiological methods were as described previously (Richmond and Jorgensen, 1999) with the following modifications. Ventral body wall muscle cells were recorded in the whole-cell voltage-clamp mode (holding potential, $-60 \mathrm{mV}$ ) using an EPC-10 patch-clamp amplifier and digitized at $2.9 \mathrm{kHz}$. Standard extracellular Ringer's solution consisted of the following (in $\mathrm{mm}$ ): $150 \mathrm{NaCl}, 5$ $\mathrm{KCl}, 5 \mathrm{CaCl}_{2}, 4 \mathrm{MgCl}_{2}, 10$ glucose, 5 sucrose, and 15 HEPES, pH 7.4 ( $\sim 340 \mathrm{mOsm})$. Calcium concentrations were lowered to 1 and $0.5 \mathrm{~mm}$, by dissecting and recording in standard Ringer's solution diluted with $0 \mathrm{~mm} \mathrm{CaCl} 2$ Ringer's. The patch pipette was filled with the following (in $\mathrm{mm}$ ): $120 \mathrm{KCl}, 20 \mathrm{KOH}, 4 \mathrm{MgCl}_{2}, 5$ $\mathrm{N}$-tris[hydroxymethyl] methyl-2-aminoethanesulfonic acid, $0.25 \mathrm{CaCl}_{2}, 4 \mathrm{Na}_{2} \mathrm{ATP}, 36$ sucrose, and 5 EGTA, pH 7.2 ( $~ 315$ mOsm). Evoked responses were stimulated with a $1 \mathrm{~ms}$ depolarizing pulse delivered via a pipette placed on the anterior ventral nerve cord. Data were acquired using Pulse software (HEKA) and subsequently analyzed and graphed using Pulsefit (HEKA), Mini Analysis (Synaptosoft), and Igor Pro (Wavemetrics).

\section{Results}

Electron tomography resolves filamentous structures within presynaptic terminals

Transmitted electron microscopy (TEM) analysis has provided critical information to decipher the structural organization of the C. elegans NMJ (Jin, 2005). The more recent implementation of the HPF technique allows instant immobilization of synaptic components in intact, behaving worms, capturing the physiologically relevant condition of the synapse (Rostaing et al., 2004; Weimer et al., 2006; Hammarlund et al., 2007). However, the $3 \mathrm{D}$ resolution of TEM is limited by the thickness of ultrathin sections, which ranges from 40 to $80 \mathrm{~nm}$, and impairs the visualization of fine structures such as filaments. To circumvent this limitation, we combined HPF and freeze substitution technology with electron tomography. We focused on the cholinergic NMJs innervating the body-wall muscles because they are relatively small, electrophysiologically accessible, and functionally well characterized. Tomo-
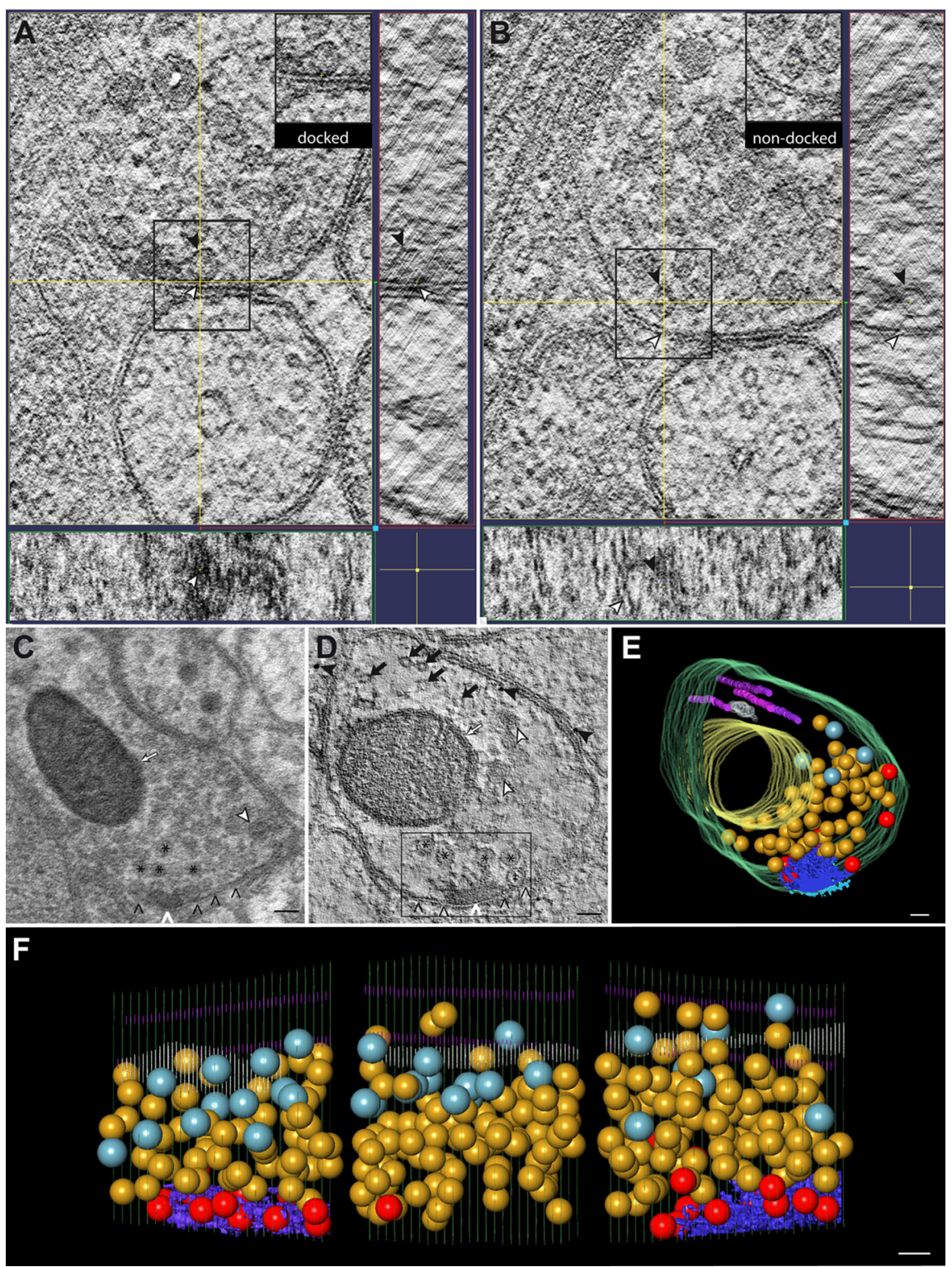

Figure 1. Electron tomography provides high 3D resolution of presynaptic boutons at a cholinergic NMJ. $A, x y / x z / y z$ representation of a tomogram at the level of a docked SV (black arrowhead). Lipid bilayers of the plasma membrane in close proximity to the SV are highlighted by white arrowhead. Section levels of the $x z$ and $y z$ dimension (smaller panels) are indicated in the $x y$ level (large panel) by yellow lines. Boxed area of region of interest at xy level is shown on the top right without annotations. $\boldsymbol{B}$, Similar representation as in $\boldsymbol{A}$ but at a level where an SV is close to the plasma membrane but not docked. C, Classical TEM micrograph of ultrathin section ( $50 \mathrm{~nm}$ thickness) of a cholinergic NMJ innervating the body-wall muscles. $\boldsymbol{D}$, Virtual slice of $\sim 1 \mathrm{~nm}$ thickness of a tomogram. Similar structures are labeled on both pictures: SVs (asterisks), docked vesicle (double cross), presynaptic cell membrane (open arrowheads), dense projections (white open arrowheads), dense-core vesicles (white arrowheads), microtubules (black arrows), and mitochondrion (white arrows). Ten nanometer gold beads (black arrowheads) were used as fiducials for generation of the tomogram shown in $\boldsymbol{D}$. Nearby virtual slice of boxed area is shown at higher magnification in Figure 3A. $\boldsymbol{E}$, Slightly tilted view of a 3D model obtained by segmentation of the 250-nm-thick tomogram shown in $\boldsymbol{D}$. Note that dense-core vesicles (brightblue) are situated at the rim of the SV pool (yellow). Docked SVs (red) touch the presynaptic membrane (green) and are mostly clustered around the dense projections (dark blue), but some docked vesicles are located distantly to the dense projections. Microtubules (violet) and smooth endoplasmic reticulum (white) are situated distant to the dense projections beyond the mitochondrion (yellow), and both structures run along the longitudinal axis of the NMJ in parallel to dense projections. Extracellular electron-dense material adjacent to dense projections is annotated in turquoise. $\boldsymbol{F}$, Side view of a 3D model of three consecutive serial tomogram sections is annotated with the same color code as $\boldsymbol{E}$. Note that the vesicle pool is spanning the interspace between dense projections (middle section). Scale bars, $50 \mathrm{~nm}$.

grams obtained from 250-nm-thick sections provided a systematic view of ultrastructural details that could not be easily resolved by standard TEM. As discussed below, the dense projections in tomograms appear as elaborated structures with filaments emanating from a core component. The lipid bilayers of 

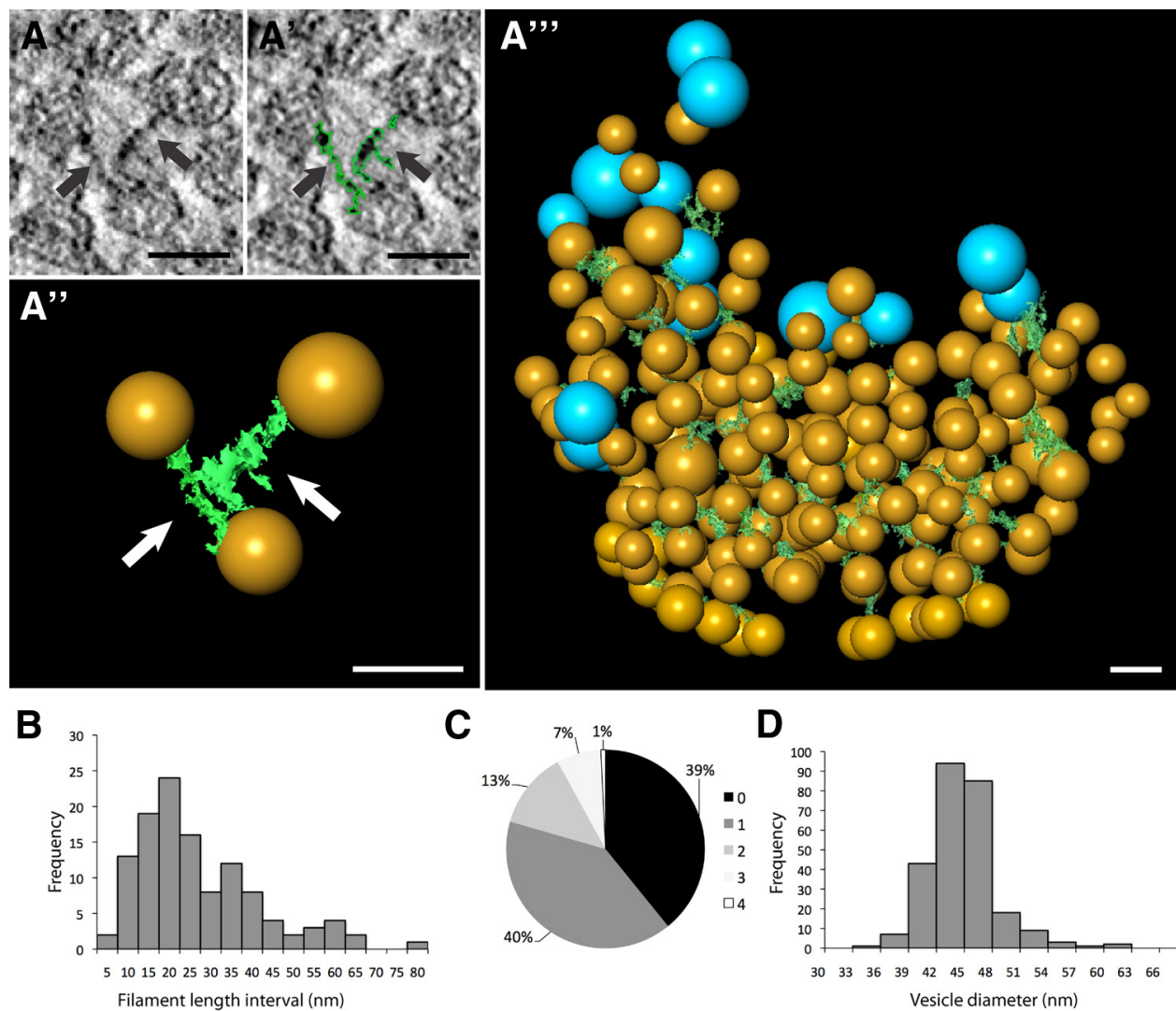

Figure 2. The presynaptic cytomatrix represents a meshwork of filaments interconnecting SVs. $A$, Virtual slice of $\sim 1 \mathrm{~nm}$ thickness with two filaments (black arrows) interconnecting three synaptic vesicles. $A^{\prime}$, Same virtual slice as in $A$ but segmentation of the two filaments outlined in green. $A^{\prime \prime}$, 3D model of the connectors shown in $A$ and $A^{\prime}$. $A^{\prime \prime \prime}$, Reconstruction of an $S V$ pool contained in a 250-nm-thick tomogram. SVs (yellow) are interconnected by filaments (green). Note that several dense-core vesicles (bright blue) are part of this network. Scale bars, $50 \mathrm{~nm}$. $\boldsymbol{B}$, Distribution of filament lengths ( $n=117,2$ tomograms). C, Distribution of number of filament contacts to adjacent vesicles (connectivity, $n=263,2$ tomograms). D, Distribution of SV diameters ( $n=263,2$ tomograms).

vesicle and plasma membranes were clearly visualized on virtual sections of the tomograms, allowing unambiguous identification of docked vesicles defined as those in direct contact with the plasma membrane (Fig. $1 A-D$ ).

For better visualization, these tomograms were segmented semiautomatically to generate $3 \mathrm{D}$ models (see Material and Methods). Figure $1 E$ provides a representative example of a presynaptic cholinergic bouton with SVs distributed around the dense projection. Vesicles contacting the plasma membrane are labeled in red. The dense-core vesicles, which most probably contain neuropeptides at cholinergic NMJs, are typically found at the outer rim of the vesicle pool (Weimer et al., 2006; Hammarlund et al., 2008). Microtubules run along the axis of the neurite and remain excluded from the pool. A long cisternae runs in parallel to the microtubule track. It may correspond to the smooth endoplasmic reticulum described previously in C. elegans neurites (Rolls et al., 2002). To gain a more holistic view of active zone organization, three consecutive tomograms of serial sections of each $250 \mathrm{~nm}$ thickness were aligned and segmented (Fig. $1 F$ ). In this example, the synaptic vesicle pool spans the space between two dense projections, resembling a synaptic "superpool" spanning several terminals as recently characterized in hippocampal neurons (Staras et al., 2010), yet docked vesicles remain concentrated around each of the dense projections. Altogether, the combination of HPF with electron tomography provides close-to-isotropic resolution of the synapse and enables comprehensive three-dimensional analysis of presynaptic structures immobilized in their physiological state.
A presynaptic filamentous network interconnects SVs at the C. elegans NMJ

At vertebrate synapses, SVs were interconnected by filamentous links called "connectors" (Landis et al., 1988; Hirokawa et al., 1989; Gustafsson et al., 2002; Siksou et al., 2007; FernándezBusnadiego et al., 2010). The combination of HPF with electron tomography identified for the first time a very similar filamentous network interconnecting synaptic vesicles in $C$. elegans NMJs (Fig. $2 A, A^{\prime}$ ). For quantification purposes, filaments were segmented semiautomatically (Fig. $2 A^{\prime}$ ) and subsequently rendered to obtain 3D models (Fig. $2 A^{\prime \prime}, A^{\prime \prime \prime}$ ). The average length of the filaments connecting vesicles within the network was $25.1 \pm$ $1.3 \mathrm{~nm}$ (mean $\pm \mathrm{SEM}, n=117$, two tomograms), with a minimum length of $3 \mathrm{~nm}$ and a maximum of $78 \mathrm{~nm}$ (Fig. 2 B). Notably, the distribution of the filament lengths does not follow a normal distribution, possibly reflecting underlying molecular heterogeneity of these filaments. Vesicle diameters ranged from 33 to $60 \mathrm{~nm}$ (Fig. $2 \mathrm{D}$ ), with an average of $41.9 \pm 0.2 \mathrm{~nm}$ (mean \pm SEM, $n=263$, two tomograms). The radial density of the vesicle population did not significantly vary in the bouton along the proximodistal axis from the AZ, in contrast to the axial variability reported for synaptosomes of the rat CNS (FernándezBusnadiego et al., 2010). The intervesicle connectivity ranged from nonconnected to four connections, one vesicle being connected on average with one other vesicle via these filaments (Fig. $2 C)$. We also observed tethers connecting clear vesicles to densecore vesicles and filaments linking vesicles and mitochondria (data not shown). 
Overall, we could visualize a filamentous network that interconnects vesicles within the synaptic bouton and provides a coherent architecture to the presynapse.

The dense projection physically contacts docked and nondocked vesicles

Electron-dense specializations of various shapes have been identified at most if not all chemical synapses and are thought to organize SVs at the AZ. However, their morphological features are highly dependent on the fixation and visualization techniques. To analyze the relationship between the dense projection and SVs at C. elegans NMJs, we segmented dense projections from tomograms (Fig. 3A). After $3 \mathrm{D}$ reconstruction, they appeared as elongated, complex structures (Fig. 3B) extending along the longitudinal axis of the neurites. As reported previously, these structures sometimes branched (Weimer et al., 2006; Hammarlund et al., 2007). Most strikingly, fine filaments emanated radially from a more compact core structure and could extend up to $100 \mathrm{~nm}$ into the synaptic bouton interior. These filaments contacted vesicles docked at the plasma membrane on each side of the dense projection and nondocked vesicles inside the presynaptic boutons (Fig. $3 B^{\prime}$ ). Intriguingly, we also observed short electron-dense projections on the extracellular side of the plasma membrane juxtaposed to the dense projection with densities sometimes crossing the bilayer of the plasma membrane (Fig. 3A). Together, these morphological features indicate that the dense projection establishes direct contacts with SVs and might play a functional role in organizing SV pools at the AZ.

\section{Docked vesicles lose close contact with dense projections in unc-10 mutants}

To test whether the observed interactions between the dense projection and SVs impose constraints on vesicle distribution, we first analyzed cholinergic NMJs of unc-10(md1117) mutants. UNC-10/RIM is an integral AZ protein, which interacts with multiple synaptic proteins and is implicated in several processes, including SV docking and priming, through interactions with RAB-3 and Munc13, respectively (Koushika et al., 2001; Schoch et al., 2002, 2006; Dulubova et al., 2005). Previous work using conventional TEM revealed that SVs contacting the plasma membrane were enriched within $\sim 100 \mathrm{~nm}$ from the dense projection. In unc-10 mutants, this population was significantly depleted (Weimer et al., 2006). To test whether the dense projection-vesicle contacts observed in tomograms of the wildtype synapses might reflect functional interactions, we compared the clustering of docked vesicles in relation to dense projections in wild-type and unc-10 mutant backgrounds (Fig. 4A,B). To quantify the clustering, we measured the $3 \mathrm{D}$ distance from the vesicle to the nearest protrusion emanating from the dense pro- jection. At wild-type synapses, $\sim 60 \%$ of the docked SVs were in direct contact with the dense projection, whereas in $u n c-10 \mathrm{mu}$ tants this fraction dropped to $\sim 20 \%$ (Fig. $4 E$ ). Overall, the distribution of the docked vesicles was significantly shifted toward a more distal position from the dense projections in $u n c-10 \mathrm{com}$ pared with wild-type synapses (Fig. 4D).

Together, these data suggest that the depletion of docked vesicles at the AZ in unc-10 mutants results from the disruption of interactions normally existing between the vesicles and dense projection filaments.

\section{SYD-2/liprin is required to localize docked vesicles at the $\mathrm{AZ}$} The UNC-10/RIM binding partner SYD-2/liprin is considered an integral dense projection component based on immuno-EM detection within the dense projections of C. elegans NMJs (Yeh et al., 2005; Weimer et al., 2006) and is thought to play a crucial role in AZ organization (Zhen and Jin, 1999; Dai et al., 2006; Patel et al., 2006). To establish how SYD-2/liprin regulates SV organization at the ultrastructural level, we analyzed cholinergic NMJs of syd-2(ju37) mutants using HPF and electron tomography. Dense projections were readily identified in the syd-2 mutants. As in unc-10 mutants, we observed a similar redistribution of docked vesicles away from the dense projection (Fig. $4 C, D$ ). In addition, the number of nondocked vesicles contacting the dense projec- 

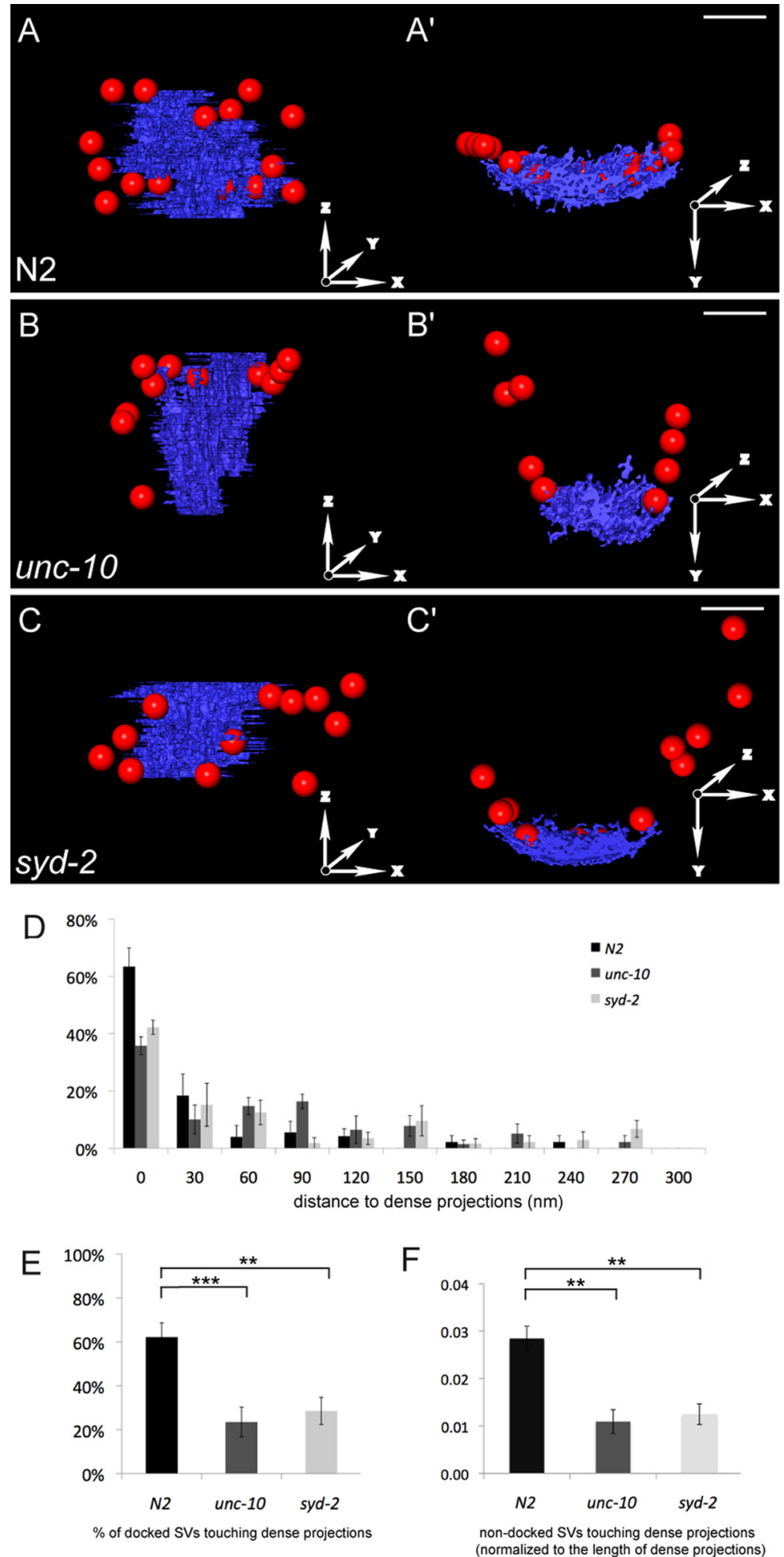

Figure 4. Docked vesicles have reduced contact with dense projections in unc-10 and syd-2 mutants. $\boldsymbol{A}$, In wild-type N2 strains, most docked vesicles (red) are in direct contact with dense projections (blue). In unc-10(md1117) (B) and syd2(ju37) (C) mutants docked vesicles localize away from dense projections. $\boldsymbol{D}$, Distribution of docked vesicles plotted in 30 $\mathrm{nm}$ intervals distance from the dense projections. The distribution of unc-10 (KS test, $D=0.4170 ; p<0.001$ ) and syd-2 (KS test, $D=0.3163 ; p=0.009$ ) is significantly different compared with N2. The distribution of unc-10 compared with syd-2 is not significantly different (KS test, $D=0.1401 ; p=0.681$ ). $\boldsymbol{E}$, Percentage of docked vesicles with direct contact to dense projections is significantly reduced in unc-10 (Fisher's exact test, $p=0.00002374$ ) and syd-2 (Fisher's exact test, $p=$ 0.001630 ) compared with N2. $\boldsymbol{F}$, The number of nondocked vesicles contacting the dense projection (normalized against the length of the dense projection) is significantly reduced in unc-10 (Fisher's exact test, $p=0.007204$ ) and syd-2 (Fisher's exact test, $p=0.009215$ ) compared with $\mathrm{N} 2, n=5$ tomograms for each genotype. Scale bars, $100 \mathrm{~nm} .{ }^{* *} p<0.01$, *** $p<0.001$. tion within the synaptic bouton was also significantly reduced (Fig. $4 F$ ).

To test whether the morphological alterations that we identified by electron tomography in $u n c-10$ and syd-2 mutants were functionally relevant, we analyzed synaptic transmission by electrophysiology. Evoked responses obtained from syd-2 mutant cholinergic NMJs in standard Ringer's solution ( $5 \mathrm{mM} \mathrm{Ca}^{2+}$ ) exhibited a $32 \%$ decrease in evoked amplitude relative to wild type ( $p=0.0003$, MannWhitney test) (Fig. 5). A similar reduction in evoked response amplitude has been reported previously for unc-10 mutants (Gracheva et al., 2008). Given that UNC$10 / \mathrm{RIM}$ is thought to facilitate both vesicle docking and calcium influx at the AZ, we next addressed the calcium dependence of syd-2 mutants. With decreasing extracellular calcium concentrations, the defect in evoked transmission became more pronounced in syd-2 mutants, with a 55\% reduction in $1 \mathrm{mM} \mathrm{Ca}^{2+}(p=0.004)$ and a $66 \%$ reduction in $0.5 \mathrm{mM} \mathrm{Ca}^{2+}(p=0.004$, Mann-Whitney test) relative to wild type (Fig. 5). In syd-2(ju37) mutants, the miniature synaptic event frequency was reduced by $53 \%$, similar to the evoked response deficit [38.9 \pm 5 events/s in syd-2(ju37) $(n=$ 12) vs $82.3 \pm 6$ events/s in wild type ( $n=$ 30); $p=0.0003$ ], whereas miniature event amplitude was unaltered [syd-2(ju37), $24.7 \pm 2.6 \mathrm{pA}$ vs wild type, $24.1 \pm 1.1 \mathrm{pA}$; NS], further supporting a presynaptic role for SYD-2 in exocytosis.

To test whether UNC-10 and SYD-2 work in linear or parallel functional pathways to tether synaptic vesicles to the dense projection and thereby regulate synapse efficiency, the evoked responses of unc10(md1117) syd-2(ju37) double mutants were analyzed. Evoked amplitudes of double mutants (Fig. 5G) showed a similar reduction and dependence on calcium concentration as unc-10 (md1117) (Fig. 5F) and syd-2(ju37) (Fig. 5D) single mutants, arguing that these two proteins regulate cholinergic synaptic transmission in a linear pathway rather than independent pathways.

Altogether, these data suggest that disruption of SYD-2/liprin prevents the recruitment of SVs at the AZ, which likely disrupts spatial coupling between primed vesicles and calcium influx during presynaptic depolarization.

\section{Discussion}

Because synaptic transmission occurs on a millisecond timescale, acquiring highresolution images of the synapse in its "native" state is challenging. The slow time course of chemical fixation, the propen- 
sity of fixatives to trigger SV release (Smith and Reese, 1980) and to distort cellular compartments through osmotic effects, represent some of the challenges in this endeavor. The implementation of HPF fixation to immobilize synaptic structures in a few milliseconds circumvents many of these technical issues. The combination of HPF with electron tomography performed on intact C. elegans, in the present study, has advanced our imaging capabilities, revealing previously uncharacterized details of the presynaptic ultrastructural organization. This allows us to observe, for the first time, a presynaptic cytomatrix in C. elegans, resembling that at vertebrate synapses. Moreover, 3D reconstruction of these presynaptic densities revealed, unexpectedly, elaborate structures with filaments contacting SVs. Ultrastructural comparison between the wild-type, syd-2/liprin, and unc-10/RIM mutants by this methodology identified a novel mechanism to both retain SVs at the $\mathrm{AZ}$ and ensure efficient coupling with calcium signaling.

Structural connections between synaptic vesicles were initially observed at vertebrate synapses using quick freezing and etching (Landis et al., 1988; Hirokawa et al., 1989). Because this technique only provides access to the first $20-30 \mathrm{~nm}$ of the tissue depth, full reconstruction of the presynaptic cytomatrix awaited electron tomography analysis. Examination of high-pressure frozen tissues either by cryo-EM (Fernández-Busnadiego et al., 2010) or after freeze substitution (Siksou et al., 2007) confirmed the existence of filaments between SVs, with an average length of $10-15 \mathrm{~nm}$ in rat cerebrocortical synaptosomes and 30 $\mathrm{nm}$ at hippocampal synapses. The average intervesicle connectivity varied from 1.5 to 2 connectors per vesicle. The cytomatrix present at the C. elegans NMJ is similar, with connectors averaging 25 $\mathrm{nm}$ in length and an intervesicle connectivity approaching 1 .

This filamentous meshwork is proposed to limit the diffusion of vesicles and maintain a vesicular pool in the presynaptic bouton (Landis et al., 1988). Genetically disrupting this structure would provide a means of testing this hypothesis, yet the molecular composition of these connectors remains elusive. Initial studies suggested that they might be composed of synapsin (Takei et al., 1995; Gitler et al., 2004). However, tomographic analysis of synapsin triple-knock-out mice found that, although vesicle number was decreased at individual boutons, the mean distance of the remaining SVs to the AZ was unchanged and filaments could still be observed between vesicles (Siksou et al., 2007). These data suggest that only a subset of filaments might be synapsin dependent, in agreement with the multi-modal distribution of connector lengths in every system so far studied, including now $C$. elegans.

At the center of the $\mathrm{AZ}$ resides the dense projection. This electron-dense specialization is remarkably tortuous, with radial filaments emanating from a longitudinal core and extending up to $100 \mathrm{~nm}$ into the interior of the synaptic bouton. The tips of these filaments contact vesicles in the bouton, as well as vesicles docked at the plasma membrane. The structure of the C. elegans dense projection resembles the presynaptic specializations present at the Drosophila NMJs. Initial studies described the Drosophila presynaptic densities as T-bars made of a pedestal and a platform, but more recent analysis using HPF has revealed much more elaborate filamentous structures at the T-bar that contact SVs (Fouquet et al., 2009; Jiao et al., 2010). These filaments likely consist of Bruchpilot, an ERC/CAST scaffold protein (Wagh et al., 2006), yet the relationship between T-bars and membranedocked vesicles remains to be analyzed after HPF, to our knowledge. Tomographic analysis of frog NMJs has also revealed the presence of ordered presynaptic densities with docked SV linked to longitudinal "beams" through interactions with short "ribs." These structures, of unknown composition, have been proposed to localize docked SVs in close proximity to voltage-dependent calcium channels (VDCCs) visualized by freeze fracture as "pegs" in the presynaptic plasma membrane (Harlow et al., 2001).

With the exception of a few specialized synapses such as the photoreceptor ribbon synapse in which ribbons likely convey vesicles to the AZ (Bunt, 1971; Gray and Pease, 1971) and tether them at the plasma membrane (Raviola and Gilula, 1975), the 
ultrastructure of most vertebrate central synapses shares little resemblance to C. elegans dense projections or Drosophila T-bars. For example, the classical presynaptic grid structure described by EM pioneers at vertebrate CNS synapses likely results from precipitation of the cytomatrix between docked synaptic vesicles by phosphotungsten acid (Gray, 1963). Recent EM tomographic analysis of chemically fixed spine synapses identified polyhedral cages that were proposed to dock vesicles at the plasma membrane (Zampighi et al., 2008). However, these structures were not identified in studies combining HPF and tomography. Analysis of rodent hippocampal synapses after HPF fixation and freeze substitution (Siksou et al., 2007, 2009) or with cryo-EM (Fernández-Busnadiego et al., 2010) identified docked SVs in contact with electron-dense material clustered at the inner face of the plasma membrane. In addition, long filaments of $\sim 80 \mathrm{~nm}$ emanated from the plasma membrane and contacted SVs in the interior of the bouton. Although these structures are morphologically diverse among vertebrate and invertebrate synapses, it is tempting to postulate that all presynaptic densities evolved from an ancestral organization already present in the "ursynapse" that was inferred from genomic paleontology (Ryan and Grant, 2009). Alternatively, different solutions might have arisen over time to fulfill specific requirements at different synapses. Testing these hypotheses will require the identification of the proteins present in these varied structures to evaluate the evolutionary conservation at the molecular level.

The C. elegans liprin ortholog SYD-2 is an integral component of the dense projection in which it was detected by immuno-EM (Yeh et al., 2005). Initial analysis of syd-2 mutants by conventional EM reported flattened and expanded dense projections at GABAergic NMJs and a broadened distribution of SVs (Zhen and Jin, 1999). Our analysis of cholinergic NMJs in syd-2 mutants after HPF fixation did not reveal dramatic disorganization of the dense projections, although they tended to look less compact with occasional ectopic patches of electron-dense material resembling small dense projections (data not shown). The differences in fixation procedures might account for some of the variability in syd-2 mutant synaptic ultrastructure, but it is also possible that the organizational role of SYD-2/liprin is more apparent at larger AZs such as those of GABA NMJs or interneuronal synapses than at the smaller AZs of cholinergic NMJs. Regardless, our analysis identified a prominent role of SYD-2 in organizing vesicle pools at the AZ. Strikingly, both the number of SV s contacted by dense projection filaments in the interior of the boutons and the number of docked vesicles contacting the dense projection were decreased in syd-2 mutants, albeit slightly less than in unc-10 mutants. The physiological correlate of this docked vesicle redistribution is an increased dependence of evoked release on extracellular calcium concentration, suggesting spatial uncoupling between primed vesicles and calcium influx during presynaptic depolarization. A similar reduction in synaptic transmission together with AZ disorganization was previously reported in Drosophila mutants for liprin- $\alpha$, although calcium dependence was not directly tested (Kaufmann et al., 2002).

A previous study using HPF and conventional TEM demonstrated that disrupting RAB-3, a small GTPase present on SVs and interacting with UNC-10/RIM, causes depletion of docked SVs close to the dense projection, very similar to the phenotype observed for unc-10 mutants (Weimer et al., 2006; Gracheva et al., 2008). In both cases, however, the total number of docked vesicles per synapse was similar to the wild type. Using tomography, we now demonstrate that both UNC-10 and its binding partner SYD-2 are required to tether vesicles to the dense projection and retain docked vesicles in close proximity. The fact that the electrophysiological defects of unc-10 syd-2 double mutants are no more severe than $u n c-10$ and syd-2 single mutants suggests that these two proteins act in the same pathway to regulate SVs. We, therefore, speculate that SYD-2 may contribute to the localization of UNC-10 at the AZ through direct and/or indirect interactions, involving proteins of the ERC family such as ELKS-1 in C. elegans and Bruchpilot in Drosophila (Ko et al., 2003; Dai et al., 2006; Fouquet et al., 2009). Interactions between UNC-10 and RAB-3 may then promote SV tethering to the dense projection, before initiating the SV docked/primed state via UNC-13 interactions, consistent with the previously characterized functional role of UNC-10 at a pre-priming step (Koushika et al., 2001). After SVs have been docked and primed, the interaction between RAB-3 and UNC-10 may restrict the lateral diffusion of these vesicles along the membrane, tethering them at the $\mathrm{AZ}$, close to VDCCs. This subset of docked SVs would then be in a privileged location, efficiently coupled to calcium influx, similar to the RAB3-dependent "super-primed" pool, previously described at vertebrate synapses (Schlüter et al., 2006).

\section{References}

Bárcena M, Koster AJ (2009) Electron tomography in life science. Semin Cell Dev Biol 20:920-930.

Bunt AH (1971) Enzymatic digestion of synaptic ribbons in amphibian retinal photoreceptors. Brain Res 25:571-577.

Couteaux R, Pécot-Dechavassine M (1970) Synaptic vesicles and pouches at the level of "active zones" of the neuromuscular junction (in French). C R Acad Sci Hebd Seances Acad Sci D 271:2346-2349.

Dai Y, Taru H, Deken SL, Grill B, Ackley B, Nonet ML, Jin Y (2006) SYD-2 Liprin- $\alpha$ organizes presynaptic active zone formation through ELKS. Nat Neurosci 9:1479-1487.

Dulubova I, Lou X, Lu J, Huryeva I, Alam A, Schneggenburger R, Südhof TC, Rizo J (2005) A Munc13/RIM/Rab3 tripartite complex: from priming to plasticity? EMBO J 24:2839-2850.

Fernández-Busnadiego R, Zuber B, Maurer UE, Cyrklaff M, Baumeister W, Lucic V (2010) Quantitative analysis of the native presynaptic cytomatrix by cryoelectron tomography. J Cell Biol 188:145-156.

Fouquet W, Owald D, Wichmann C, Mertel S, Depner H, Dyba M, Hallermann S, Kittel RJ, Eimer S, Sigrist SJ (2009) Maturation of active zone assembly by Drosophila Bruchpilot. J Cell Biol 186:129-145.

Gitler D, Takagishi Y, Feng J, Ren Y, Rodriguiz RM, Wetsel WC, Greengard P, Augustine GJ (2004) Different presynaptic roles of synapsins at excitatory and inhibitory synapses. J Neurosci 24:11368-11380.

Gracheva EO, Hadwiger G, Nonet ML, Richmond JE (2008) Direct interactions between $C$. elegans RAB-3 and Rim provide a mechanism to target vesicles to the presynaptic density. Neurosci Lett 444:137-142.

Gray E, Pease H (1971) On understanding the organisation of the retinal receptor synapses. Brain Res 35:1-15.

Gray EG (1963) Electron microscopy of presynaptic organelles of the spinal cord. J Anat 97:101-106.5.

Gustafsson JS, Birinyi A, Crum J, Ellisman M, Brodin L, Shupliakov O (2002) Ultrastructural organization of lamprey reticulospinal synapses in three dimensions. J Comp Neurol 450:167-182.

Hammarlund M, Palfreyman MT, Watanabe S, Olsen S, Jorgensen EM (2007) Open syntaxin docks synaptic vesicles. PLoS Biol 5:e198.

Hammarlund M, Watanabe S, Schuske K, Jorgensen EM (2008) CAPS and syntaxin dock dense core vesicles to the plasma membrane in neurons. J Cell Biol 180:483-491.

Harlow ML, Ress D, Stoschek A, Marshall RM, McMahan UJ (2001) The architecture of active zone material at the frog's neuromuscular junction. Nature 409:479-484.

Hirokawa N, Sobue K, Kanda K, Harada A, Yorifuji H (1989) The cytoskeletal architecture of the presynaptic terminal and molecular structure of synapsin 1. J Cell Biol 108:111-126.

Jiao W, Masich S, Franzén O, Shupliakov O (2010) Two pools of vesicles associated with the presynaptic cytosolic projection in Drosophila neuromuscular junctions. J Struct Biol 172:389-394.

Jin Y (2005) Synaptogenesis. WormBook 16:1-11. 
Jin Y, Garner CC (2008) Molecular mechanisms of presynaptic differentiation. Cell Dev Biol 24:237-262.

Kaufmann N, DeProto J, Ranjan R, Wan H, Van Vactor D (2002) Drosophila Liprin- $\alpha$ and the receptor phosphatase Dlar control synapse morphogenesis. Neuron 34:27-38.

Ko J, Na M, Kim S, Lee JR, Kim E (2003) Interaction of the ERC family of RIM-binding proteins with the liprin-alpha family of multidomain proteins. J Biol Chem 278:42377-42385.

Koushika SP, Richmond JE, Hadwiger G, Weimer RM, Jorgensen EM, Nonet ML (2001) A post-docking role for active zone protein Rim. Nat Neurosci 4:997-1005.

Kremer JR, Mastronarde DN, McIntosh JR (1996) Computer visualization of three-dimensional image data using IMOD. J Struct Biol 116:71-76.

Landis DM, Hall AK, Weinstein LA, Reese TS (1988) The organization of cytoplasm at the presynaptic active zone of a central nervous system synapse. Neuron 1:201-209.

McDonald KL, Auer M (2006) High-pressure freezing, cellular tomography, and structural cell biology. BioTechniques 41:137-141.

Patel MR, Lehrman EK, Poon VY, Crump JG, Zhen M, Bargmann CI, Shen K (2006) Hierarchical assembly of presynaptic components in defined $C$. elegans synapses. Nat Neurosci 9:1488-1498.

Raviola E, Gilula NB (1975) Intramembrane organization of specialized contacts in the outer plexiform layer of the retina. A freeze-fracture study in monkeys and rabbits. J Cell Biol 65:192-222.

Richmond JE, Jorgensen EM (1999) One GABA and two acetylcholine receptors function at the C. elegans neuromuscular junction. Nat Neurosci 2:791-797.

Robertson JD (1987) The early days of electron microscopy of nerve tissue and membranes. In: International review of cytology. Overviews: thirtyfive years of cell biology (Jeon KW, ed), pp 129-201. San Diego: Academic.

Rolls MM, Hall DH, Victor M, Stelzer EH, Rapoport TA (2002) Targeting of rough endoplasmic reticulum membrane proteins and ribosomes in invertebrate neurons. Mol Biol Cell 13:1778-1791.

Rostaing P, Weimer RM, Jorgensen EM, Triller A, Bessereau JL (2004) Preservation of immunoreactivity and fine structure of adult C. elegans tissues using high-pressure freezing. J Histochem Cytochem 52:1-12.

Ryan TJ, Grant SG (2009) The origin and evolution of synapses. Nat Rev Neurosci 10:701-712.

Schlüter OM, Basu J, Südhof TC, Rosenmund C (2006) Rab3 superprimes synaptic vesicles for release: implications for short-term synaptic plasticity. J Neurosci 26:1239-1246.

Schoch S, Gundelfinger ED (2006) Molecular organization of the presynaptic active zone. Cell Tissue Res 326:379-391.

Schoch S, Castillo PE, Jo T, Mukherjee K, Geppert M, Wang Y, Schmitz F, Malenka RC, Südhof TC (2002) RIM1 $\alpha$ forms a protein scaffold for regulating neurotransmitter release at the active zone. Nature 415: 321-326.

Schoch S, Mittelstaedt T, Kaeser PS, Padgett D, Feldmann N, Chevaleyre V, Castillo PE, Hammer RE, Han W, Schmitz F, Lin W, Südhof TC (2006) Redundant functions of RIM1alpha and RIM2alpha in $\mathrm{Ca}^{2+}$-triggered neurotransmitter release. EMBO J 25:5852-5863.
Siksou L, Rostaing P, Lechaire JP, Boudier T, Ohtsuka T, Fejtová A, Kao HT, Greengard P, Gundelfinger ED, Triller A, Marty S (2007) Threedimensional architecture of presynaptic terminal cytomatrix. J Neurosci 27:6868-6877.

Siksou L, Varoqueaux F, Pascual O, Triller A, Brose N, Marty S (2009) A common molecular basis for membrane docking and functional priming of synaptic vesicles. Eur J Neurosci 30:49-56.

Smith JE, Reese TS (1980) Use of aldehyde fixatives to determine the rate of synaptic transmitter release. J Exp Biol 89:19-29.

Staras K, Branco T, Burden JJ, Pozo K, Darcy K, Marra V, Ratnayaka A, Goda Y (2010) A vesicle superpool spans multiple presynaptic terminals in hippocampal neurons. Neuron 66:37-44.

Sudhof TC (2004) The synaptic vesicle cycle. Annu Rev Neurosci 27: 509-547.

Takei Y, Harada A, Takeda S, Kobayashi K, Terada S, Noda T, Takahashi T, Hirokawa N (1995) Synapsin I deficiency results in the structural change in the presynaptic terminals in the murine nervous system. J Cell Biol 131:1789-1800.

Wagh DA, Rasse TM, Asan E, Hofbauer A, Schwenkert I, Dürrbeck H, Buchner S, Dabauvalle MC, Schmidt M, Qin G, Wichmann C, Kittel R, Sigrist SJ, Buchner E (2006) Bruchpilot, a protein with homology to ELKS/ CAST, is required for structural integrity and function of synaptic active zones in Drosophila. Neuron 49:833-844.

Wang X, Hu B, Zieba A, Neumann NG, Kasper-Sonnenberg M, Honsbein A, Hultqvist G, Conze T, Witt W, Limbach C, Geitmann M, Danielson H, Kolarow R, Niemann G, Lessmann V, Kilimann MW (2009) A protein interaction node at the neurotransmitter release site: domains of Aczonin/Piccolo, Bassoon, CAST, and Rim converge on the N-terminal domain of Munc13-1. J Neurosci 29:12584-12596.

Weimer RM (2006) Preservation of C. elegans tissue via high-pressure freezing and freeze-substitution for ultrastructural analysis and immunocytochemistry. In: Methods in molecular biology (351): C. elegans (Strange K, ed), pp 203-221. Totowa, NJ: Humana.

Weimer RM, Gracheva EO, Meyrignac O, Miller KG, Richmond JE, Bessereau JL (2006) UNC-13 and UNC-10/Rim localize synaptic vesicles to specific membrane domains. J Neurosci 26:8040-8047.

White JG, Southgate E, Thomson JE, Brenner S (1986) The structure of the nervous system of the nematode Caenorhabditis elegans. Philos Trans $\mathrm{R}$ Soc Lond B Biol Sci 314:1-340.

Yeh E, Kawano T, Weimer RM, Bessereau JL, Zhen M (2005) Identification of genes involved in synaptogenesis using a fluorescent active zone marker in Caenorhabditis elegans. J Neurosci 25:3833-3841.

Zampighi GA, Zampighi L, Fain N, Wright EM, Cantele F, Lanzavecchia S (2005) Conical tomography II: a method for the study of cellular organelles in thin sections. J Struct Biol 151:263-274.

Zampighi GA, Fain N, Zampighi LM, Cantele F, Lanzavecchia S, Wright EM (2008) Conical electron tomography of a chemical synapse: polyhedral cages dock vesicles to the active zone. J Neurosci 28:4151-4160.

Zhai RG, Bellen HJ (2004) The architecture of the active zone in the presynaptic nerve terminal. Physiology 19:262-270.

Zhen M, Jin Y (1999) The liprin protein SYD-2 regulates the differentiation of presynaptic termini in C. elegans. Nature 401:371-375. 